\title{
Nurse's Perception of Barriers to Research Utilization in Hospitals; Comparative Descriptive Study
}

\author{
Imad H. Fashafsheh ${ }^{1}$, Ahmad Ayed ${ }^{2}$, Jafer A. Mohammed ${ }^{3}$, Yasir A. Alotaibi ${ }^{1}$ \\ ${ }^{1}$ Nursing Department, University of Bisha, Bisha, KSA \\ ${ }^{2}$ Nursing, Arab American University, Jenin, Palestine \\ ${ }^{3}$ Dentistry and Nursing, Alfarabi Colleges of Medicine, Riyadh, KSA \\ Email: ^fashafsheh@gmail.com
}

How to cite this paper: Fashafsheh, I.H., Ayed, A., Mohammed, J.A. and Alotaibi, Y.A. (2020) Nurse's Perception of Barriers to Research Utilization in Hospitals; Comparative Descriptive Study. Open Journal of Nursing, 10, 1-14.

https://doi.org/10.4236/ojn.2020.101001

Received: September 24, 2019

Accepted: January 4, 2020

Published: January 7, 2020

Copyright $\odot 2020$ by author(s) and Scientific Research Publishing Inc. This work is licensed under the Creative Commons Attribution International License (CC BY 4.0).

http://creativecommons.org/licenses/by/4.0/

\begin{abstract}
Background: Although the number of studies on research utilization is steadily growing, but still there is a strong need to identify the main causes that inhibit the use of research findings in the clinical setting. Aim of the Study: To assess nurse's perception of barriers to research utilization in Saudi Arabia and Palestine Hospitals. This study focuses on medical and surgical nurses in private hospitals. Method: A convenient sample of 156 medical nurses, from 6 general private hospitals, 3 from north Palestine and 3 from Riyadh City (KSA). Results: Out of 180 nurses employed from private hospitals, a total of 156 questionnaires were returned (response rate 86.67\%). Results in a final sample size for analysis of 156 . The demographic characteristics, age, gender, hospital, role on health care team, clinical experience, marital status, education degree, and current certified in a special area of respondents are shown in Table 1 . Their mean age was 29.41, about half of them below 6-year clinical experience 77 (49.4\%). Although around $90(57.7 \%)$ of the participants from Palestine hospitals and 66 (42.3\%) from Saudi Arabia hospitals, almost half of them their education (diploma) 85 (54.5\%). In addition, 114 (73.1\%) were female, 85 (54.5\%) have current certified in a special area, and $140(89.7 \%)$ respondents worked as staff nurses. Regarding perceptions of barriers to research utilization, the results of the barriers show in the total scale a mean of 3.15 out of $5 \mathrm{f}(\mathrm{SD}=0.55$; $95 \% \mathrm{CI}$ 3.05 to 3.23). The subscales of setting and research were the highest one (mean $=3.22, \mathrm{SD}=(0.63)$, and the lowest one is "nurse" (mean $=2.95, \mathrm{SD}=$ 0.75). Conclusions: The main barriers of research utilization in practice for nurse are nurses themselves, the setting in the hospitals and research in its process and findings.
\end{abstract}




\section{Keywords}

Nurses, Perception, Research Utilization, Barriers

\section{Introduction}

Research is a field that always there is new information rising [1]. Research utilization is the process of synthesizing, disseminating and using research to make changes in nursing practice and can be used to support clinical decision-making [2].

Apart from the efforts of research evolution aim at bridging the gap between theory and clinical practice, the translation of nursing knowledge into practice is called research utilization [3].

Research utilization continually expresses concern about whether nurses use the available research evidence to guide their clinical practice. Researchers are gaining into process of diffusion and utilization of new knowledge through, innovation and utilization and disseminate research findings to potential users including researchers and clinicians [4].

Many health care settings push for the development of evidence-based practice, because most health care providers do not apply basic sciences and clinical research [5].

Evidence-based practice was described as integration of individual clinical expertise with the best available external expertise, clinical expertise is skills that clinician develops over time through practice and experience. The ultimate goal of evidence-based practice is to use the best quality of knowledge to provide a greater impact on patient's health [6].

Nurses may have a positive attitude towards research-based practice, but to transfer evidence of research into practice remains remain slow [7]. This due to possible factors influencing nurses to successfully transfer and implemented research evidence in practice such as lack of financial support, staff shortage, poor communication and over dependent of nurses on physicians in making decision about patient care [8]. Insufficient time on the job to implement research findings and inadequate time to read research considered as primary barrier [9].

There is often a gap between theoretical knowledge and it is applied upon practical area [10]. Evidence-based practice and research utilization are concepts related to the identification, utilization and application of knowledge from research findings to clinical practice [11]. The focus should be upon manipulating factors at work place as well as learning environment that leads to less cost of health care and decrease patients suffering [12].

Research environment should contain the development of policies such as guidelines, clinical setting protocols and procedural manual based on the best available nursing information [13]. Literature showed several factors that facilitate nurses to utilize research findings; those factors mainly related to nurse's 
competencies in understanding and applying research and other factors related to administration, resources for research design \& sample [14]. Literature also showed three common barriers lack of time, lack of authority and language barriers. Some barriers are related to language like the use of English language in research publication, which is a barrier to none English population besides, lack of coordination between education institutions and clinical settings [15] [16].

There is a complex set of skills required to utilize research findings, for nurses reading habits; most of clinical nurses read journal monthly [17], even though most of the nurses practices are based on their experiences and not on research findings [13], while Australians nurses likely to read research journal usually, and Turkish nurses attended conferences at least once per year. Nursing research publication is not widely read by clinical nurses [18] there is a real barrier between nurses' attitude and research knowledge, sometimes, the main barrier is nurses' negative attitude towards research [19], they have inadequate knowledge about research utilization, this due to absent or limited of basic research knowledge [20].

In more than 10 studies conducted in research utilization in practice, organization support was cited as top barriers [21] besides, insufficient authority to change in practice [4].

Among registered nurses, the top three barriers were "inability to do research "lack of time to conduct research and apply finding of research [10]. Another study showed the top barriers to research utilization were "lack of access to research expertise lack of physicians support to change practice and lack of time for research [22]. Individual factors like beliefs, attitude, socio-demographic and professional characteristic may affect research utilization [19], statistical knowledge and appraisal of research findings cited as the most common personal barriers [23]. Literature also mentions other individual factors as, lack of training, lack of incentive especially in terms of career development, influence of opinion leaders and lack of motivation [23]. Lack of organization support is considered as the most common barriers to research utilization in many recent studies [21] [24]. Barriers related to organization setting include team, diffusion roles, poor leadership and absent of appropriate performance measurements [25], besides lack of nurses' authority which is consider as the top greatest barrier [26], and they need collaboration among manager's academics and practitioners to overcome this barrier. Limitation of financial support leads difficulty in applying the research findings which usually needs materials and equipment [27], in general, many factors face organization setting as lack of support or incentive for clinical practice development, poor multi-professional teamwork and poor access to research or data base access [28].

Significance of the study:

Despite the spread of nursing research, there is still poor utilization of research findings into clinical practice [29]. From our experiences and clinical observation in privates Hospitals in Saudi Arabia (Riyadh) and Palestine nurses still keep in their previous experiences and practice, which is not based on new 
research findings or evidence-based practice which is considered a major factor influence nursing care.

Aim of study: To assess nurse's perception of barriers to research utilization in Saudi Arabia and Palestine Private Hospitals in medical surgical departments.

Research questions:

1) Is their relationship between experience of nurses and research utilization in practice?

2) Is their relationship between educational level and research utilization in practice?

3) Is their relationship between setting in the hospitals and research utilization in practice?

4) Is their relationship between administrations or management type in the hospitals and research utilization in practice?

\section{Methodology}

\section{Design and sampling:}

This descriptive comparative study was carried out with 156 nurses from private hospitals in Palestine and Saudi Arabia between June-September 2017. The data collection instrument used for the study consisted of the Barriers to Research Utilization Scale with 31 items, and a self-administered questionnaire form that contained demographic data, educational background and nurses' involvement in research activities.

\section{Sample:}

Out of 180 questionnaires distributed 156 responses, the response rate was $86.67 \%$, with convenience sample of 156 registered nurses, 90 of whom are Palestinian from governmental hospitals and 66 from selected private hospitals in from Riyadh Kingdom of Saudi Arabia.

The $\mathrm{G}^{*}$ power version 3.0.10 used to estimate necessary sample size. Using a calculated medium effect size of 0.3 for $\mathrm{f}$-test to determine the difference between means of the groups, an alpha of 0.05 , and power of 0.95 based on the assumption of an expected difference resulted in a sample of 180 participants. To overcome the attrition rate and who apologizes to participate, the final sample was 156 participants.

Inclusion criteria: All staff nurses in the general and critical care departments.

Exclusion criteria: Practical nurses and staff nurses with less than 3-months experience.

\section{Tool:}

The data was collected using a modified Barriers to Research Utilization Scale with 31 items (Barriers Scale) which is the most widely used standard of measurement for the nurses. The questionnaire sent to five experts for evaluation, comments on item 5 in the first category (Nurse) and on items 4 and 11 in the second category (Setting). 
The Barriers Scale was originally developed in the USA in the 1980s and modified by Funk et al. (32).

Questionnaires of 31 items of research utilization barriers were distributed to the nurses. The barriers were categories into three categories: setting related barriers, research related barriers and nurses' related barriers.

\section{Procedure:}

After getting the formal approval from ministry of health $(\mathrm{MOH})$ and the administration of private hospital from Riyadh city (KSA), we started distributing and collecting our research questionnaire to different hospitals. The study was conducted on two phases; In first phase short interview conducted with nurses to assess their attitude to research utilization and explore research utilization in clinical practice, in second phase distribution of the questionnaire and explaining the content of questionnaire to the nurses as well as explained its main purpose in a simple and clear way, after that we asked them to answer all the questions. Data collected and data entered into the computer in order to analyze.

\section{Ethical consideration:}

Every participant in the study received an explanation about the purpose confidentiality of the study. Participation in the study was voluntary and all data and information's gathered is strictly confidential and is not to be accessed by any other without prior permission from the participants, moreover, the participant had the right to withdraw at any time if he or she can't complete the questionnaire.

\section{Results}

The aim of this study was to assess nurse's perception of barriers to research utilization in Saudi Arabia and Palestine Private Hospitals in medical surgical departments.

Results in a final sample size for analysis of 156 . The demographic characteristics, age, gender, hospital, role on health care team, clinical experience, marital status, education degree, and current certified in a special area of respondents are shown in Table 1. Their mean age was 29.41, about half of them 77 (49.4\%) below 6 years' clinical experience. In addition, 114 (73.1\%) were female, 85 (54.5\%) have current certified in a special area, and140 (89.7\%) respondents worked as staff nurses.

Regarding perceptions of barriers to research utilization, the results of the Barriers Scale and its 3 subscales are shown in Table 2. The scores ranged from 1 to 5 , with a mean of 3.15 for the overall score ( $\mathrm{SD}=0.55$; $95 \%$ CI 3.05 to 3.23 ). The subscales of setting and research were the highest one (mean $=3.22, \mathrm{SD}=$ $(0.63)$, and the lowest one is "nurse" (mean $=2.95, \mathrm{SD}=0.75$ ).

The top 10 items rated as a great or moderate barrier for research utilization, 3 were setting-related, 5 were nurse-related, and 2 were research-related. "The nurse is unwilling to change/try new ideas" ( $\mathrm{n}=149 ; 93.1 \%$ ); "The nurse is uncertain of whether to believe the results of the research" ( $\mathrm{n}=149,93.1 \%)$; "The 
Table 1. The participants' demographic characteristic, $(\mathrm{N}=156)$.

\begin{tabular}{|c|c|}
\hline Variable & N (\%) \\
\hline Age (years) & Mean (SD): 29.41 (8.84) \\
\hline \multicolumn{2}{|l|}{ Gender } \\
\hline Male & $42(26.9)$ \\
\hline Female & $114(73.1)$ \\
\hline \multicolumn{2}{|l|}{ Hospital } \\
\hline Palestine hospitals & $90(57.7)$ \\
\hline Saudi Arabia hospitals & $66(42.3)$ \\
\hline \multicolumn{2}{|l|}{ Current role on health care team } \\
\hline Staff nurse & $140(89.7)$ \\
\hline Administrator & $2(1.3)$ \\
\hline Nursing Supervisor & $2(1.3)$ \\
\hline Other & $12(7.7)$ \\
\hline \multicolumn{2}{|l|}{ Clinical experience (years) } \\
\hline $1-5$ years & $77(49.4)$ \\
\hline $6-10$ years & $47(30.1)$ \\
\hline $11-15$ years & $14(9.0)$ \\
\hline More than 15 years & $18(11.5)$ \\
\hline \multicolumn{2}{|l|}{ Marital status } \\
\hline Single & $64(41.0)$ \\
\hline Married & $92(59.0)$ \\
\hline \multicolumn{2}{|l|}{ Education degree } \\
\hline Diploma & $85(54.5)$ \\
\hline Bachelor & $69(44.2)$ \\
\hline Master & $2(1.3)$ \\
\hline \multicolumn{2}{|l|}{ Current certified in a special area } \\
\hline Yes & $85(54.5)$ \\
\hline No & $71(45.5)$ \\
\hline
\end{tabular}

Table 2. Means and Standard Deviations of the BARRIERS Scale and its subscales $(\mathrm{N}=$ 156).

\begin{tabular}{ccc}
\hline Scale or subscales & Mean (SD) & $95 \% \mathrm{CI}$ \\
\hline BARRIERS Total Scale & $3.15(0.55)$ & $3.05-3.23$ \\
Nurse & $2.95(0.75)$ & $2.83-3.05$ \\
Setting & $3.22(0.63)$ & $3.12-3.32$ \\
Research & $3.22(0.63)$ & $3.12-3.32$ \\
\hline
\end{tabular}

nurse does not have time to read research" ( $n=146 ; 91.3 \%)$; "The nurse does not see the value of research for practice" ( $n=141,88.1 \%)$; "The nurse does not feel capable of evaluating the quality of the research" ( $\mathrm{n}=141,88.1 \%)$; “The nurse feels the benefits of changing practice will be minimal" ( $n=141,88.1 \%)$; "The nurse feels results are not generalizable to own setting" ( $n=141,88.1 \%)$; "The nurse feels results are not generalizable to own setting" ( $n=139,86.9 \%)$; “The nurse sees little benefit for self" ( $n=137,85.7 \%)$; “Other staffs are not supportive of implementation" ( $n=137,85.7 \%)$; and "Research report/articles are not published fast enough" $(\mathrm{n}=136,85.0 \%)$ as shown in Table 3.

Table 3 shows the results for the 31 individual items of the BARRIERS Scale 
and their rank order. The top one was pertaining to the "Setting," while the bottom two items were all pertaining to the "Nurse."

\section{Comparisons of groups of nurses:}

Significant statistical results were found for different subgroups of nurses in Table 4:

1) Different scientific degrees resulted in statistically significant differences in scores of both the total scale $(f=(3.152)=7.76, p<0.001)$ and subscales (the

Table 3. Barriers items in rank order $(\mathrm{N}=156)$.

\begin{tabular}{|c|c|c|c|}
\hline Items & Subscale & $\begin{array}{c}\text { Rating item as moderate } \\
\text { or great barrier (\%) }\end{array}$ & Rank order \\
\hline Insufficient time on the job to implement new ideas. & Setting & $96(61.5)$ & 1 \\
\hline Researchers are written in English. & Research & $95(60.9)$ & 2 \\
\hline The facilities are inadequate for implementation. & Setting & $82(52.6)$ & 3 \\
\hline The nurse does not have time to read research. & Setting & $72(46.2)$ & 4 \\
\hline Other staffs are not supportive of implementation. & Setting & $71(45.5)$ & 5 \\
\hline The amount of research information is overwhelming. & Research & $71(45.5)$ & $5^{*}$ \\
\hline There is not a documented need to change practice. & Setting & $69(44.2)$ & 6 \\
\hline The nurse does not see the value of research for practice. & Nurse & $67(42.9)$ & 7 \\
\hline The nurse is uncertain of whether to believe the results of the research. & Nurse & $67(42.9)$ & $7^{\star *}$ \\
\hline The relevant literature is not compiled in one place. & Setting & $67(42.9)$ & $7^{\star *}$ \\
\hline The nurse feels that results are not generalizable to own setting. & Setting & $65(41.7)$ & 8 \\
\hline Research report/articles are not clearly and readably. & Research & $65(41.7)$ & $8^{*}$ \\
\hline The nurse is unaware of research. & Nurse & $64(41.0)$ & 9 \\
\hline Implications for practice are not made clear. & Setting & $64(41.0)$ & $9^{* *}$ \\
\hline The conclusions drawn from the research are not justified. & Research & $64(41.0)$ & $9^{* *}$ \\
\hline Research has not been replicated. & Research & $60(38.5)$ & 10 \\
\hline Literature reports had conflict results. & Research & $60(38.5)$ & $10^{*}$ \\
\hline The nurse feels the benefits of changing practice will be minimal. & Nurse & $59(37.8)$ & 11 \\
\hline Research report/articles are not published fast enough. & Research & $59(37.8)$ & $11^{\star *}$ \\
\hline The conclusions drawn from the research are not justified. & Research & $59(37.8)$ & $11^{\star *}$ \\
\hline The nurse does not feel she/he has authority to change patient care procedures. & Setting & $56(35.9)$ & 12 \\
\hline The research has methodological inadequacies. & Research & $54(34.6)$ & 13 \\
\hline The nurse is isolated from knowledgeable with whom to discuss research. & Nurse & $53(34.0)$ & 14 \\
\hline The nurse sees little benefit for self. & Nurse & $51(32.7)$ & 15 \\
\hline Administration will not allow implementation. & Setting & $51(32.7)$ & $15^{* * *}$ \\
\hline The research is not relevant to the nurse's practice. & Research & $51(32.7)$ & $15^{\star * *}$ \\
\hline Statistical analyses are not understandable. & Research & $51(32.7)$ & $15^{* * *}$ \\
\hline The nurse is isolated from colleagues with whom to discuss the research. & Nurse & $50(32.1)$ & 16 \\
\hline Physicians will not cooperate with implementation. & Setting & $45(28.8)$ & 17 \\
\hline The nurse is unwilling to change/try new ideas. & Nurse & $39(25.0)$ & 18 \\
\hline The nurse feels not capable of evaluating the quality of the research. & Nurse & $32(20.5)$ & 19 \\
\hline
\end{tabular}

*Two items had the same percent ranking. ${ }^{*}$ Three items had the same percent ranking. ${ }^{* * *}$ Four items had the same percent ranking. 
Table 4. Factors influencing perceptions of barriers to research $(\mathrm{N}=156)$.

\begin{tabular}{|c|c|c|c|c|c|}
\hline \multicolumn{4}{|c|}{ Highest degree obtained } & \multicolumn{2}{|c|}{ Hospital } \\
\hline & Diploma & Bachelor & Master & Palestine hospitals & Saudi Arabia hospitals \\
\hline & \multicolumn{3}{|c|}{ Mean (SD) } & \multicolumn{2}{|c|}{ Mean (SD) } \\
\hline \multirow[t]{2}{*}{ Barrier total scale } & $3.30(0.52)$ & $2.97(0.54)$ & $2.82(0.39)$ & $3.15(0.51)$ & $3.14(0.61)$ \\
\hline & \multicolumn{3}{|c|}{$F=7.76, p=0.001$} & \multicolumn{2}{|c|}{$\mathrm{t}=0.10, \mathrm{p}=0.924$} \\
\hline \multirow[t]{2}{*}{ Nurse } & $3.17(0.71)$ & $2.66(0.66)$ & $3.39(0.55)$ & $2.91(0.67)$ & $3.00(0.81)$ \\
\hline & \multicolumn{3}{|c|}{$\mathrm{F}=10.96, \mathrm{p}=0.000$} & \multicolumn{2}{|c|}{$\mathrm{t}=0.76, \mathrm{p}=0.450$} \\
\hline \multirow[t]{2}{*}{ Setting } & $3.32(0.55)$ & $3.10(0.70)$ & $3.00(0.39)$ & $3.26(0.60)$ & $3.17(0.66)$ \\
\hline & \multicolumn{3}{|c|}{$\mathrm{F}=2.58, \mathrm{p}=0.079$} & \multicolumn{2}{|c|}{$\mathrm{t}=0.92, \mathrm{p}=0.361$} \\
\hline \multirow[t]{2}{*}{ Research } & $3.11(0.59)$ & $2.85(0.58)$ & $1.95(0.19)$ & $3.08(0.56)$ & $3.09(0.67)$ \\
\hline & \multicolumn{3}{|c|}{$\mathrm{F}=7.13, \mathrm{p}=0.001$} & \multicolumn{2}{|c|}{$\mathrm{t}=0.02, \mathrm{p}=0.984$} \\
\hline
\end{tabular}

$\mathrm{P}<0$.

range of from 2.82 to 3.30). After pair wise comparison, scientific degrees of Diploma had higher scores compared with those of Bachelor and with Master in both the total scale and two subscales (Setting and Research). Nurses with scientific degree of Master achieved higher scores than those with Diploma and Bachelor in "Nurse" subscales, the lowest scientific degree the higher the barriers' scores. The results indicate that scientific degree is a significant factor influencing perceptions of barriers.

2) Compared with Nurses from different hospitals, those from Palestine hospital or Saudi Arabia hospitals hadn't significant differences of both the total scale $(t=(155)=0.10, p<0.924)$ and subscales (Nurse, Setting, and Research). This indicates that no differences between Palestine hospitals and Saudi Arabia hospitals towards Nurse's perception of barriers to research.

\section{Discussion}

The results of this study suggest that there should be a comprehensive short and long-term strategy to promote research utilization in the subscales of the study (Nurse, Setting, and Research) to overcome barriers that negatively affects or inhibit the application of research results in practice.

The present study consists of 156 registered nurses that were investigated about their knowledge and perception of research barberries, response rate was $86.67 \%)$. The demographic characteristics include, age, gender, hospital, role on health care team, clinical experience, marital status and education degree. Their mean age was 29.41, about half of them below 6 years clinical experience77 (49.4\%). In addition, 114 (73.1\%) were female, 85 (54.5\%) had current certified in a special area, and $140(89.7 \%)$ respondents worked as staff nurses.

Individual factors like beliefs, attitude, socio-demographic and professional characteristic may affect research utilization [19], statistical knowledge and appraisal of research findings cited as the most common personal barriers [23]. 
The recent study showed that the more experience the less application and awareness of research utilization and more barriers perceived of this group. This goes with the results of the study titled (barriers in implementing research among registered nurses working in the care of the elderly: a multicenter study in Spain) [30], nurses who obtained their nursing degree more than 10 years ago was in had higher level of barriers that the newer generations who received more training and awareness of research through the curricula offered by their nursing schools, and this result was shown in another study by [31].

Level of education goes with level of barriers in this study the higher level of education the less barriers of research utilization, this result congruent with the study done by Eui Geum Oh (2008) on critical care Kurian nurses in which nurses with higher level of education are welling more to apply research article results [32].

Regarding barriers to research utilization for this study, the result showed the five top barriers are related to the setting, the most common subscale is, insufficient time on job to implement new research idea this goes with these results [10] [22] [33]. This barrier also coincided with the most frequently reported barrier in similar studies [30] [34] [35].

The facilities are inadequate for implementation and there no documentation needed to change practice compare to other studies the most common research barriers were also related to organization setting [7] [13] [23]. Lack of time is the major barrier related to organization setting, this may be due to shortage in the numbers of nursing staff particularly in private hospitals, and the nurses are under pressure and workload [10].

The results of the current study showed that there is some consistency across studies regarding how nurses perceive barriers in different countries and the barriers identified by nurses did not seem influenced by culture, or other factors. The barriers remained relatively stable over time

\section{Conclusion}

Findings of this study suggest the necessity of nurses regular reading of the research articles, the higher level of searching skill on research documents, a clear understanding of evidence-based nursing practice (EBNP), beliefs and, particularly, high level of professional autonomy. These findings highlight the importance of educational preparation and provide fundamental information to health care managers and administrators in developing actual educational programs for nurses working in clinical settings and at the same time to support nurses in their efforts to change according to research findings. However, more studies about this important issue are needed on a regular basis trying to minimize the barriers.

\section{Limitations}

The findings limited in terms of generalizing beyond the sample due to our use 
of non-probability sampling, others were not fully considerate of organizational characteristics as function and structure, which are actually different in both countries and cultures. Actually, some cultures hinder professional autonomy and evidence-based nursing practice implementation.

\section{Conflicts of Interest}

The authors declare no conflicts of interest regarding the publication of this paper.

\section{References}

[1] Athanasakis, E. (2013) Nurses' Research Behavior and Barriers to Research Utilization into Clinical Nursing Practice: A Closer Look. International Journal of Caring Sciences, 6, 16-28.

[2] Burns, N. and Grove, S. (2005) The Practice of Nursing Research: Conduct, Critique and Utilization. 4th Edition, W.B. Saunders, Philadelphia.

[3] Yava, A., Tosun, N., Ciçek, H., Yavan, T., Terakye, G. and Hatipoğlu, S. (2009) Nurses' Perceptions of the Barriers to and the Facilitators of Research Utilization in Turkey. Applied Nursing Research, 22, 166-175. https://doi.org/10.1016/j.apnr.2007.11.003

[4] Fink, R., Thompson, C.J. and Bonnes, D. (2005) Overcoming Barriers and Promoting the Use of Research in Practice. Journal of Nursing Administration, 35, 121-129. https://doi.org/10.1097/00005110-200503000-00005

[5] Brook, R., McGlynn, E. and Shekelle, P. (2000) Defining and Measuring Quality of Care: A Perspective from US Researchers. International Journal for Quality in Health Care, 12, 281-295. https://doi.org/10.1093/intqhc/12.4.281

[6] Sackett, D., Rosenburg, W., Gray, J., Haynes, R. and Richardson, W. (1996) Evidence-Based Medicine: What It Is and What It Isn't.

[7] Chien, W.T. (2013) Nurses' Perceived Barriers to and Facilitators of Research Utilization in Mainland China: A Cross-Sectional Survey. Open Nursing Journal, 7, 96-106. https://doi.org/10.2174/1874434601307010096

[8] French, B. (2005) Contextual Factors Influencing Research Use in Nursing. Worldviews Evidence Based Nursing, 2, 172-183. https://doi.org/10.1111/j.1741-6787.2005.00034.x

[9] Mohammadkarim, B., Mehdi, R., Ramin, R. and Behzad, M. (2016) Barriers to the Application of the Research Findings from the Nurses' Perspective: A Case Study in a Teaching Hospital. Journal of Education and Health Promotion, 5, 14. https://doi.org/10.4103/2277-9531.184553

[10] Mehradad, N., Salsali, M. and Kazemnejad, A. (2008) The Spectrum of Barriers to and Facilitators of Research Utilization in Iranian Nursing. Journal of Clinical Nursing, 17, 2194-2202. https://doi.org/10.1111/j.1365-2702.2007.02040.x

[11] Bussières, A., et al. (2016) Evidence-Based Practice, Research Utilization, and Knowledge Translation in Chiropractic: A Scoping Review. BMC Complementary and Alternative Medicine, 16, 216. https://doi.org/10.1186/s12906-016-1175-0

[12] Al Ghabeesh, S. (2015) Barriers and Suggested Facilitators to the Implementation of Best Practice: An Integrative Review. Open Journal of Nursing, 5, 77-87.

[13] Oh, E. (2008) Research Activities and Perceptions of Barriers to Research Utilization among Critical Care Nurses in Korea. Intensive and Critical Care Nursing, 24, 
314-322. https://doi.org/10.1016/j.iccn.2007.12.001

[14] Veeramah, V. (2004) Utilization of Research Findings by Graduate Nurse and Midwives. Journal of Advanced Nursing, 47, 183-191. https://doi.org/10.1111/j.1365-2648.2004.03077.x

[15] Latifi, S., Khalilpour, A., Rabiee, O. and Amani, N. (2012) Barriers to Research Utilization among Clinical Nurse. Journal of Mazandaran University of Medical Sciences, 22.

[16] Heydari, A. and Zeydi, A. (2014) Barriers to and Facilitators of Research Utilization among Iranian Nurses: A Literature Review. Journal of Caring Sciences, 3, 265-275.

[17] Retsas, A. (2000) Barriers to Using Research Evidence in Nursing Practice. Journal of Advanced Nursing, 31, 599-606. https://doi.org/10.1046/j.1365-2648.2000.01315.x

[18] Uysal, A., Temel, A.B., Ardahan, M. and Ozkahraman, S. (2010) Barriers to Research Utilization among Nurses in Turkey. Journal of Clinical Nursing, 19, 3443-3452. https://doi.org/10.1111/j.1365-2702.2010.03318.x

[19] Squires, J.E., Estabrooks, C.A., Gustavsson, P. and Wallin, L. (2011) Individual Determinants of Research Utilization by Nurse: A Systematic Review Update. Implementation Science, 6, 1. https://doi.org/10.1186/1748-5908-6-1

[20] Mottola, C.A. (1996) Research Utilization and the Continuing/Staff Development Educator. The Journal of Continuing Education in Nursing, 27, 168-175.

[21] Bryar, R.M., et al. (2003) The Yorkshire Barriers Project: Diagnostic Analysis of Barriers to Research Utilization. International Journal of Nursing Studies, 40, 73-84. https://doi.org/10.1016/S0020-7489(02)00039-1

[22] Chien, W.T. (2010) A Survey of Nurses' Perceived Barriers to Research Utilization in Hong Kong. Journal of Clinical Nursing, 19, 3584-3586.

[23] Hutchinson, A.M. and Johnston, I. (2004) Bridging the Divide: A Survey of Nurses' Opinions Regarding Barriers to, and Facilitators of, Research Utilization in the Practice Setting. Journal of Clinical Nursing, 13, 304-315. https://doi.org/10.1046/j.1365-2702.2003.00865.x

[24] Rogers, E. (2003) Diffusion of Innovation. 5th Edition, Free Press, New York, London, Toronto, Sydney, Singapore.

[25] Kitson, A., Ahmed, L.B., Harvey, G., Seers, K. and Thompson, D.R. (1996) From Research to Practice: One Organizational Model for Promoting Research-Based Practice. Journal of Advanced Nursing, 23, 430-440. https://doi.org/10.1111/j.1365-2648.1996.tb00003.x

[26] Wang, L.-P., Jiang, X.-L., Wang, L., Wang, G.-R. and Bai, Y.-J. (2013) Barriers to and Facilitators of Research Utilization: A Survey of Registered Nurses in China. PLoS ONE, 8, e81908. https://doi.org/10.1371/journal.pone.0081908

[27] El-Badawy, A. and Kassam, I. (2008) Nurses Perception of Barriers and Facilitates of Research Utilization in the Clinical Setting. Bulletin of Alexandria Faculty of Medicine, 44, 811-820.

[28] Le May, A., Alexander, C. and Mulhall, A. (1998) Research Utilization in Nursing: Barriers and Opportunities. Journal of Clinical Effectiveness, 3, 59-63. https://doi.org/10.1108/eb020875

[29] Majid, S., et al. (2011) Adopting Evidence-Based Practice in Clinical Decision Making: Nurses' Perceptions, Knowledge, and Barriers. Journal of the Medical Library Association, 99, 229-236. https://doi.org/10.3163/1536-5050.99.3.010

[30] Sarabia-Cobo, et al. (2015) Barriers in Implementing Research among Registered 
Nurses Working in the Care of the Elderly: A Multicenter Study in Spain. Applied Nursing Research, 28, 352-355. https://doi.org/10.1016/j.apnr.2015.03.003

[31] Boström, M., Kajermo, N., Nordström, G. and Wallin, L. (2008) Barriers to Research Utilization and Research Use among Registered Nurses Working in the Care of Older People: Does the Barriers Scale Discriminate between Research Users and Nonresearch Users on Perceptions of Barriers? Implementation Science, 3, 24. https://doi.org/10.1186/1748-5908-3-24

[32] Oh, E.G. (2008) Research Activities and Perceptions of Barriers to Research Utilization among Critical Care Nurses in Korea. Intensive and Critical Care Nursing, 24, 314-322. https://doi.org/10.1016/j.iccn.2007.12.001

[33] Funk, S., Champagne, M., Wiese, R. and Tornquist, E. (1991) The Barriers to Research Utilization Scale. Applied Nursing Research, 4, 39-45.

https://doi.org/10.1016/S0897-1897(05)80052-7

[34] Moreno-Casbas, T., Fuentelsaz-Gallego, C., Gonzalez-Maria, E. and Gil de Miguel, A. (2010) Barreras para la utilización de la investigación. Estudio descriptivo en profesionales de enfermería de la práctica clínica y en investigadores activos. Enfermería Clínica, 20, 153-164. https://doi.org/10.1016/j.enfcli.2010.01.005 http://www.sciencedirect.com/science/article/pii/S113086211000015X

[35] Kang, Y. and Yang, I.-S. (2016) Evidence-Based Nursing Practice and Its Correlates among Korean Nurses. Applied Nursing Research, 31, 46-51.

https://doi.org/10.1016/j.apnr.2015.11.016 


\section{Appendix}

\section{Dear colleague nurses}

Could you please give us a few minutes of your time to participate in our study about nurse's perception of barriers to research utilization in hospitals?

This study is being conducted by Dr. Imad Fashafsheh and his research committee. The purpose of this study is to identify the barriers of research utilization perceived by nurses. Your participation in this study is voluntary and you are free to withdraw your participation any time, and be sure that there are no risks associated with your participation.

While you will not experience any direct benefits from participation, information collected in this study may benefit the nursing care and patient's health promotion.

The survey collects no identifying information of any respondents or institutions. All of the response in the survey will be recorded anonymously.

By completing and submitting this survey, you are indicating your consent to participate in the study. Your participation is appreciated.

This questionnaire contains two parts: the first part is demographic data, and the second part is barriers to research utilization related questions, which contain 3 subparts :

1) Nurse related questions (9 questions)

2) Setting related questions (11 questions)

3) Research related questions (11 questions)

Please feel free to select the appropriate choice (Ranked from strongly agree to strongly don't agree).

We thank you for your valuable time and effort.

\section{Research team}

Questionnaire Sheet for nurse's perception of barriers to research utilization in hospitals

\section{Part One: Demographic Data}

Please put a tick mark $(\sqrt{ })$ next to your choice on the following items:

1) Age in year:

2) Gender: Male $\square$ Female

3) Current role on health care team: Staff nurse $\square$ Administrator $\square$ Nursing Supervisor $\square$ Other

4) Experience as a registered nurse/year:

5) Marital status: Single $\square$ Married

6) Highest degree obtained: Diploma $\square$ Bachelor (BSN) $\square$ Master (MSN) $\square$.

7) Hospital: Palestine hospitals $\square$ Saudi Arabia hospitals

8) Current Certified in a special area: Yes $\square$ No $\square$

Part two: Barriers to Research Utilization

Please put a tick mark $(\sqrt{ })$ next to your choice on the following items: 


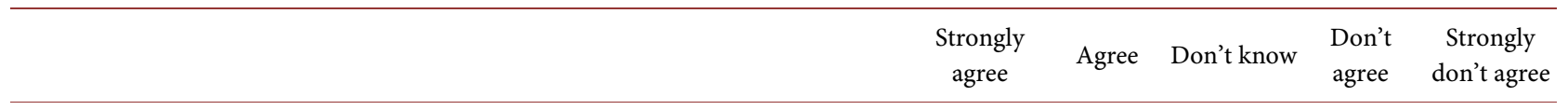

\section{1-Nurse}

1) The nurse is unaware of research.

2) The nurse is isolated from colleagues with whom to discuss the research.

3) The nurse does not see the value of research for practice.

4) The nurse is unwilling to change/try new ideas.

5) The nurse does not feel capable of evaluating the quality of the research.

6) The nurse feels the benefits of changing practice will be minimal

7) The nurse is uncertain of whether to believe the results of the research

8) The nurse sees little benefit for self

9) The nurse is isolated from knowledgeable with whom to discuss research

\section{2-Setting}

1) Insufficient time on the job to implement new ideas.

2) The nurse does not have time to read research.

3) The nurse does not feel she/he has authority to change patient care procedures.

4) The nurse feels results are not generalizable to own setting.

5) The facilities are inadequate for implementation.

6) Other staffs are not supportive of implementation.

7) Physicians will not cooperate with implementation.

8) Administration will not allow implementation.

9) Implications for practice are not made clear.

10) The relevant literature is not compiled in one place.

11) There is not a documented need to change practice.

\section{3-Research}

1) Research report/articles are not clearly and readably.

2) Research report/articles are not published fast enough.

3) Research has not been replicated.

4) Literature reports had conflict results.

5) The research has methodological inadequacies.

6) The conclusions drawn from the research are not justified.

7) The amount of research information is overwhelming.

8) Researches are written in English.

9) The research is not relevant to the nurse's practice.

10) Statistical analyses are not understandable.

11) The conclusions drawn from the research are not justified. 